\title{
Career and Recognition of Community Health Professionals Graduated from Alioune Diop University in Bambey (Senegal)
}

\author{
Ousseynou $\mathrm{Ka}^{1,}{ }^{,}$, Fatou Oumar Ndiaye $\mathrm{Sy}^{1}$, Mountaga Elimane Dia ${ }^{1}$, Ndeye Fatou Ngom ${ }^{2}$, \\ Abdoul Aziz Ndiaye ${ }^{1}$, Aladji Madior Diop ${ }^{3}$, Boubacar Gueye ${ }^{1}$, Cheikh Tacko Diop ${ }^{1}$, Martial Coly Bop ${ }^{1}$, \\ Alioune Badara Tall ${ }^{1}$, Papa Gallo Sow ${ }^{1}$, Mansour Faye ${ }^{1}$, Lamine Guéye ${ }^{4}$ \\ ${ }^{1}$ Community Health Department, Health and Sustainable Development Training and Research Unit, Alioune Diop University, Bambey, \\ Senegal \\ ${ }^{2}$ Médecine Department, Health and Sustainable Development Training and Research Unit, Alioune Diop University, Bambey, Senegal \\ ${ }^{3}$ Sustainable Development Department, Health and Sustainable Development Training and Research Unit, Alioune Diop University, Bambey, \\ Senegal \\ ${ }^{4}$ Physiology Department, Faculty Médicine, Pharmacy and Odontology, Cheikh Anta Diop University, Dakar, Senegal
}

\section{Email address:}

ousseynou.ka@uadb.edu.sn (O. Ka)

${ }^{*}$ Corresponding author

\section{To cite this article:}

Ousseynou Ka, Fatou Oumar Ndiaye Sy, Mountaga Elimane Dia, Ndeye Fatou Ngom, Abdoul Aziz Ndiaye, Aladji Madior Diop, Boubacar Gueye, Cheikh Tacko Diop, Martial Coly Bop, Alioune Badara Tall, Papa Gallo Sow, Mansour Faye, Lamine Guéye. Career and Recognition of Community Health Professionals Graduated from Alioune Diop University in Bambey (Senegal). Central African Journal of Public Health. Vol. 7, No. 3, 2021, pp. 102-110. doi: 10.11648/j.cajph.20210703.13

Received: February 20, 2021; Accepted: May 5, 2021; Published: May 27, 2021

\begin{abstract}
Introduction: Constituting a new body to contribute to improving the health of populations, community health professionals trained at Alioune Diop University in Bambey (Senegal) must integrate into a medicalized health system where curative medicine occupies a place preponderant. Objectives: to appreciate the level of understanding of the concept of community health and the challenge of integrating graduates in community health into the health care system. Methods: This was a qualitative study conducted from February 1 to April 15, 2020, targeting community health professionals and employers. Two tools were used: semi-structured interviews for employers and the focus group for community health professionals. Results: Community health professionals are assimilated by healthcare professionals and by patients to community health workers, although the latter have no professional qualification. The actions used by employers are more akin to public health than to community health. Community health professionals reported the existence of jurisdictional conflicts within the core team of the medical region or health district. The community health professionals greatly appreciated the establishment of the community health program, which according to it should be extended to hospitals and urban populations. Moreover, they would like their recruitment into the public service to strengthen the human resources of the health system. Conclusion: Recommendations have been formulated for an appropriation of this concept by healthcare professionals and decision-makers.
\end{abstract}

Keywords: Community Health, Public health, Community Health Professionals, Community Health Workers, Jurisdictional Conflicts, Primary Health Care

\section{Introduction}

Based on the observation that prevention was insufficiently taken into account in the health care system and that in terms of health nothing can be done without the community, the academic authorities of the Ministry of Higher Education, Research and Innovation from Senegal decided to set up a diploma course in community health at the Alioune Diop University in Bambey in 2007 [1]. Their concern was to diversify the training offer through innovative 
training that meets the needs of populations and the job market. These community health professionals should not be confused with "CHW" community health workers even if there are similarities in their activities. A community health worker "ASC" is a member of the community chosen by the latter, who has received basic training to promote health or to practice certain health care, without being a health professional. [2, 3]. Moreover, it is the MSAS which defines the promotional, preventive, curative and re-adaptive activities to be entrusted to the CHWs and these can only be implemented under the supervision of qualified health workers (doctors, nurses, midwives, etc.). On the other hand, community health professionals like paramedics (nurses, midwives, etc.) benefit from diploma training and work for and with the community. This unique training in the West African region aims at putting community health professionals on the labor market capable, through a participatory approach, of identifying a priority health problem in a community, of providing suitable solutions and of evaluating the interventions carried out. Thus, community health $[4,5]$ is defined as an approach to solving health problems based on the participation and engagement of communities. This approach based on the support of enlightened public opinion and active cooperation from communities need a transfer of knowledge and skills from community health professionals to these communities so that they can play and fully assume their role. The end is their empowerment $[6,7]$. This training fills a gap in qualified human resources, since community health activities were "insufficiently" carried out by doctors specializing in public health and by paramedics from the public sector. Indeed, the latter were more concerned with administrative tasks and patient care than with promotional and preventive health activities. These graduates in community health, holders of bachelor's or master's degrees generally recruited in the health sector, most often work at the level of health districts (public sector) but also at the level of non-governmental organizations "NGOs". This desire to adopt a community approach [8] by our health authorities in solving health problems was reinforced by the creation in 2012, at the Ministry of Health and Social Action "MSAS", of a community health unit [9]. Thus, this study was conducted among community health professionals and their employers with the objective of assessing the level of understanding of the concept of community health and the challenge of integrating health graduates into the health care system.

\section{Method}

\subsection{Study Framework}

The community health system follows the pyramidal architectural organization of the Senegalese health system made up of three levels [10]. At the central level, there is the community health unit which is housed at the level of the General Directorate of Health of the MSAS. She had to develop strategic documents such as the National
Community Health Policy "PNSC" and the National Strategic Plan for Community Health "PSNSC" The objective of this PNSC is to contribute to the reduction of morbidity and mortality with full participation of communities. While the PSNSC, a tool for implementing the PNSC, aims to systematize, organize and coordinate all community interventions throughout the national territory. At the intermediate level, the medical region which is the level of supervision and coordination at the regional level of community health activities. The medical region is headed by a chief physician specializing in public health, supported by the "ECR" regional team, including among others a community health supervisor. At the peripheral level, the health district which is a level of execution of community health activities. The district is an operational zone comprising at least one health center, health posts and sometimes health huts. The district is also headed by a doctor specializing in public health supported by the district management team "ECD" including among others a focal point in community health. The function of EDC or EDC is administrative and financial management, supervision, staff training, planning, monitoring and evaluation of health activities in the region or district. Community activities benefit from financial and logistical support from civil society organizations and development partners. At the formal level, these activities are planned in the same way as the activities of health centers and posts through the operational plans of local communities. [10].

\subsection{Type of Study}

This is a study based on a qualitative approach conducted during the period from February 1 to March 15, 2020.

\subsection{Study Population}

It focused on two targets: community health graduates integrated into a professional environment and their employers.

\subsection{Collection}

Data's: The data was collected by two community health professionals with a master's degree, one of whom is enrolled in a doctorate. They work in non-governmental organizations "NGOs" and have professional experience in data collection. The interviews were recorded using a Dictaphone and then transcribed after collection. The working language was French. Two tools were used: semi-structured interviews for employers and the focus group for community health professionals.

\subsection{Collection Tools}

Interviews were conducted in a face-to-face relationship or by telephone. For each group of actors, an interview guide was designed, then tested and validated.

An interview guide for community health professionals on the themes: concept of community health, difficulties encountered during the professional career, assessment of the 
implementation of so-called community health programs.

An interview guide for employers (public sector or NGOs) on: contribution and usefulness of these community health professionals in the healthcare system.

\subsection{Data Collection}

\subsubsection{Semi-structured Interviews}

They concerned those in charge of the health system at the central, regional and peripheral level of the Ministry of Health and Social Action "MSAS" but also NGOs or one or more community health professional (s). They are also a target of great importance insofar as they are the users of the products offered by the UFR SDD. They are the most apt to appreciate the contribution of these health professionals that they use. In addition, they can make proposals in case of revision of curricula so that training can meet the needs of the labor market. Appointments were made with these officials by phone call, facilitated by the director of the UFR. They were also informed of the objectives of the interview. Interviews averaging thirty to forty-five times were conducted over the telephone and were all recorded with their consent. Those responsible were selected by consensus by teachers from the community health department according to the objectives of the study. Thus, a representative sampling was carried out by choosing a manager for each level of the health pyramid and two NGO managers. [11]. The choice of the person in charge is based on his seniority or professional experience, the number of community health professionals under his supervision, his availability, and his involvement during the development of the training model in community health. These interviews focused on the usefulness and contribution of community health professionals in health system policies and programs at both strategic and operational levels. A total of five interviews were conducted and they involved three officials from the Ministry of Health (one responsible for the community health unit, one regional doctor, and one district doctor) and two heads of NGOs.

\subsubsection{Focus Group}

The focus group concerned community health professionals whether they have a license and / or a master's degree, working in the public or private sector. They are the first target especially as they are the main ones concerned. Thus, interviewing them allows them to make an in-depth analysis of their professional career by highlighting the various problems they have been confronted with. They form a group homogeneous in relation to their training and profession due to the sampling by homogenization [11]. The selection was guided by the pedagogical manager of the UFR SDD according to the objectives of the study. The choice of professionals was based on criteria such as the employer's status, place of practice, years of experience, gender, availability, type of activities carried out, position held. The focus group facilitator was also a community health professional who had to facilitate focus groups during his professional activities. The same was true of the observer taking notes. These professionals were contacted by telephone by the teaching manager of the UFR and all gave their consent to participate. In addition, he explained to them the expected objectives during this meeting. He made a telephone call back the day before the day scheduled for the focus group. The meeting place was set at the Dakar branch of Alioune Diop University in Bambey, easy to access and known to all. Three focus groups each comprising eight professionals were organized. In each group, there were also many professionals working in the public sector and in the private sector in particular NGOs. Each focus group lasted one hour and forty-five. The three focus groups took place successively during the day on a Saturday morning (rest day). After the focus groups, a summary was carried out with the participants, followed by comments on the topic. The attendance sheet filled in by the participants included age, sex, place of exercise, position held, date of obtaining the diploma, date of recruitment and professional seniority. Three focus groups each comprising eight professionals were organized. In each group, there were also many professionals working in the public sector and in the private sector in particular NGOs. Each focus group lasted one hour and fortyfive. The three focus groups took place successively during the day on a Saturday morning (rest day). After the focus groups, a summary was carried out with the participants, followed by comments on the topic.

The number of professionals was 24 with as many men as women; they had a minimum of two years experience. These professionals worked both at the MSAS level and at the NGO level. At the MSAS level, they were divided between central, intermediate and operational levels.

\subsection{Analysis}

The content analysis was carried out by a teacherresearcher specializing in sociology and officiating at the level of the community health department of the UFR Santé et Développement Durable "SDD" since 2012. She has proven experience in qualitative research and teaches this topic constituent element for License 3 and Master 1 students from the same department. This analysis consisted initially in extracting verbatim transcriptions, the fragments or block of meaning most representative of the position of the person or group, also taking into account the facilitator's notes. After reading the interviews and focus group, the verbatim of the participants were classified in code according to their meaning in relation to the objective. The codes were then grouped manually into thematic categories established by the authors. An evolving thematic analysis, allowing an inductive approach to raw data, was carried out as the groups were carried out by the facilitator, using qualitative analysis techniques.. The analysis of the content of the speeches illustrated by the words, put in quotation marks, of the people surveyed was carried out.

\subsection{Ethics}

Participation in the survey was purely voluntary and data collection was subject to rules of confidentiality 
(anonymization of recordings and transcribed data).

\section{Results}

\subsection{Misunderstanding of the Concept of Community Health}

Community health workers said that during the internships paramedics (nurses and midwives) equated them to community health workers and they felt frustrated. This is how they were challenged on the following questions: "After the baccalaureate, why do you want to be a community health worker, what is community health, do we need studies to do health?" community said a nurse in charge of a health district". It even happened that those in charge of health structures, in particular health posts, did not know what role to entrust to them during the impregnation courses in the care environment, as a community health professional testified. "When I went to do my internship in a health, the head nurse let me know that I had no place there because he did not know the reasons why his health post was chosen as the place of internship." Thus, healthcare professionals entrusted them with tasks dedicated to community health workers (making the bed, asepsis, weight and temperature gain, sale of consultation tickets, etc.). In addition, the patients also did not understand why they were in the health structure. They called them for consultations or for treatment because they wore white coats. Moreover, the patients were always astonished that we answered that we were not trained to provide care "If you cannot consult what you are doing in the health structure, here we are sick and we need to be treated said a woman who came for consultation". A community health professional testifies "Where things go wrong is the name of the word community health. At meetings with partners when everyone introduces themselves and you say that you are in community health. Sometimes it bothers you. I think we need to review this name of community health. Because when we talk about community health it refers to community health workers. It is in this sense that I speak of embarrassment". Another community health professional corroborates this argument "We should also change the name of community health. What is currently blocking us is to say community health. When you are asked what training do you do? As soon as you say the word community health you have to argue. If you don't explain, I assure you will be taken for a community health worker". A community health professional participant stressed that he experienced difficulties before he was integrated into the medical team "The medical team at the health structure did not know what community health was and wondered on our contribution, I was asked if I had my place there and what I could do. At the beginning no task was given to me because the management team was there and everyone had their program to manage. It was later that the Chief Physician asked me to coordinate the reports of the various activities". Community health professionals specified that they were obliged before their integration into the medical team to decline their skills to the person in charge of the health structure, that is to say what they know how to do "From my first day of service to the health district, I spoke with my district chief medical officer for 2 hours of time. I explained to him what community health was, he understood and was convinced. And systematically he entrusted me as a first task, the responsibility of the epidemiological surveillance of the Expanded Program of Vaccination". Despite the incomprehension aroused by the concept of "community health" by healthcare professionals, community health professionals did not regret being community health professionals "I have had to travel with quite a few doctors and they ask me if I am a doctor. I tell them that I am proud to be a community health professional". Moreover, medical professionals (regional and district doctors) as well as NGO leaders appreciate the work of community health professionals within their respective structures. They emphasize that these community health professionals play an important role in achieving the objectives of disease control programs (malaria, tuberculosis, AIDS, neglected tropical diseases, etc.). Indeed their training, oriented on preventive and promotional activities, they can complement paramedics more accustomed to care. Testimonies from district chief medical officers and NGO managers illustrate the importance of the place of these community health professionals in the health system: "The paramedical will take care of care and the community health professional will take care of prevention and promotional activities which will relieve the paramedics. Thus having this pair in a health structure, in particular a health post, will further improve the health of the populations, affirmed a district doctor "-" Now, in our recruitment, we favor community health professionals since they are well equipped to lead the desired activities, ie promotional and preventive health activities. Whereas other time when we recruit an agent we are obliged to strengthen his skills so that he is operational said an NGO manager""Thanks to our community health focal point who is a community health professional, we are managed to raise the vaccination indicators and now I have entrusted him with the popularization of mutual health organizations and the adherence of the populations to them.

\subsection{Community Health Approach or Public Health?}

Some community health professionals asserted that they do more public health than community health "We apply the directives of our employers to the communities said a participant" - "In what we do, the population is not at the heart of the strategies, it is not even involved in decisionmaking. So it's anything but community health in our opinion but public health affirmed a participant". For others, projects depend on external funding and there is almost no community ownership. Generally, in the preparation of project documents, for which the financing period is imperatively limited, no chapter is conceived and drawn up anywhere which provides for the sustainability of the achievements in terms of support at the end of the project budget plan, human and material. The testimonies of community health professionals are illustrative in this regard: 
"In an NGO where I was recruited for a three-year project; as soon as the partners withdrew, the project stopped and yet it was very beneficial for the populations"-"When the NGOs were in locality X from 2010 to 2015 , all the indicators were green, the health huts were functional. But when they left, the community actors no longer being motivated, the indicators began to pose a problem, so there is no community ownership of activities and, moreover, in a project logic, we do not even imagine that actions can be carried out without external funding ". "In an NGO where I was recruited for a three-year project; as soon as the partners withdrew, the project stopped and yet it was very beneficial for the populations"-"When the NGOs were in locality X from 2010 to 2015 , all the indicators were green, and the health huts were functional. But when they left, the community actors no longer being motivated, the indicators began to pose a problem, so there is no community ownership of activities and, moreover, in a project logic, we do not even imagine that actions can be carried out without external funding ". "In an NGO where I was recruited for a three-year project; as soon as the partners withdrew, the project stopped and yet it was very beneficial for the populations"-"When the NGOs were in locality X from 2010 to 2015, all the indicators were green, and the health huts were functional. But when they left, the community actors no longer being motivated, the indicators began to pose a problem, so there is no community ownership of activities and, moreover, in project logic, we do not even imagine that actions can be carried out without external funding". The project stopped and yet it was very beneficial for the populations"-"When the NGOs were in locality X from 2010 to 2015 , all the indicators were green, the health huts were functional. But when they left, the community actors no longer being motivated, the indicators began to pose a problem, so there is no community ownership of activities and, moreover, in project logic, we do not even imagine that actions can be carried out without external funding". The project stopped and yet it was very beneficial for the populations"-"When the NGOs were in locality X from 2010 to 2015 , all the indicators were green, the health huts were functional. But when they left, the community actors no longer being motivated, the indicators began to pose a problem, so there is no community ownership of activities and, moreover, in a project logic, we do not even imagine that actions can be carried out without external funding".

According to community health professionals, it is important to separate community health from community participation. They specified that: "Involving the community in the project or using community health workers does not mean community health, which translates the community's commitment from the identification of needs to the assessment, it is is beyond community participation ". This approach is supported by the words of an NGO manager "We are funded by external partners for activities validated by the Ministry of Health and their implementation is done in concert with the district team, however we let us inform and involve local populations".

\subsection{The Emergence of Jurisdictional Conflicts}

Supervisors (medical region) or focal points (health district level) of health programs think that we want to take their place, affirms a community health professional: "During internship, we sometimes come into conflict with the supervisor of health activities. health education because he thinks we criticize his work and yet we only seek to improve it". "When the head of the primary health care service went on leave, I carried out the activities to the satisfaction of the district chief medical officer, when he returned he did not tell me anything more." At the level of the health district managerial team, there is sometimes a conflict of competences between the community health focal point and that of primary health care. "When I was appointed community health focal point.

Community health professionals have observed that community health activities at the level of the health district or medical region are generally entrusted to health care workers, most often nurses after a field experience. "My insertion in the district was difficult because it was the "health education (EPS)" focal point which at the same time acted as the community health focal point; which meant that I was in the service for a year without doing anything. Moreover, on my note of affection, I was considered a community health actor and I was under the supervision of the PE focal point. I was not invited to coordination meetings It was when the EPS focal point took leave that I began to be integrated and subsequently the managers of the health structure began to invite me to the meetings of the health district executive team and so it is that I was able to carry out activities, to the satisfaction of the management team; now I am well integrated.

\subsection{Community Health Policy}

Community health professionals expressed their satisfaction that Senegal now has a community health policy and strategic plan, thus demonstrating the authorities' willingness to involve the community in health programs. "It is good that Senegal has took the initiative to set up a community health policy; it's good anyway and I think that we are ahead of some countries of the sub-region since I represented our NGO in sub-regional and African meetings affirmed a participant who has capitalized on nine years of experience ". Community health professionals have judged that the strategic plan drawn up by the Ministry of Health and Social Action is more oriented towards huts, health posts and rural populations. However, they recognize that community health should also concern the entire health pyramid and urban populations; this point of view was expressed in these terms: "We must extend community health interventions to the level of hospitals but also to urban populations; we can be in Dakar and do community health.

Community health professionals have underlined that compared to other bodies (doctors, nurses, midwives, etc.), the level of recruitment of community health graduates by the public service is currently low. However, our authorities 
are aware of the importance of prevention and promotional activities in the fight against diseases "prevention is better than cure" especially in a context of scarcity of financial resources and the emergence of chronic non-communicable diseases but also diseases with epidemic potential. Moreover, with the occurrence of epidemic or even pandemic diseases (Ebola, Coronavirus, etc.), national and international health authorities have stressed the importance of community involvement and community health professionals have been constantly called upon to carry out awareness-raising, information and communication activities with the communities, as well as their commitment. Indeed, the role of these professionals is to involve the population in the management of their own health, which makes it possible to strengthen social ties and develop the feeling of having a say in the development of the group (empowerment). "We are community health professionals and we are ready to serve the health system everywhere, community health professionals said." In addition, they underline that they are not integrated into the device during the development of the strategic community health plan. "In the strategic community health plan, there is no place or role and function of professionals in this discipline, this plan is more intended for community health workers".

Community health professionals stressed the need to expand the job description of the supervisor or community health focal point at the level of the medical region or the health district. "I think that the activities of supervisors or focal points" primary health care and "health education" are almost similar to those of the "community health" supervisor or focal point, so for efficient management of human resources, these 3 positions must be merged and this new position assigned to community health professionals. I am not preaching for our corporation, but I can say that we are better equipped to manage these activities since the appointment of nurses to these positions depends more on their experience than on their training. Community health professionals deemed it necessary to have a framework for consultation and advocacy like the other sister corporations. "Doctors are protected by their union as are midwives and nurses; we too must have a framework for reflection and advocacy to defend the interests of our corporation".

These community health professionals noted that preventive activities are not valued by the more curativeoriented care system. These comments are illustrative: "When you make a home visit, it is not mentioned anywhere, it is an activity, I would say invisible, whereas all the curative activities of care professionals, in particular morbidity, are listed in the registers of consultation"-"You can know at district level or regional or national level the number of people with malaria or tuberculosis or deaths... but is this the case for preventive and promotional activities and yet that's also important ".

Community health professionals have advocated, like the National Council for the Fight against AIDS "CNLS" or the Cell for the Fight against Malnutrition "CLM" that the community health unit be institutionally better repositioned or set up as an institution, department or agency to further translate this political will since it is a transversal discipline. A community health professional affirms: "Community health does not only concern the ministry of health and social action but also the CMU, local communities, the ministry of community development, the delegation for social protection...".

\section{Discussion}

\subsection{Misunderstanding of the Concept of Community Health}

Community health workers were equated with community health workers (CHWs). Indeed, a community health worker is not a health professional; he does not have any qualifications, especially as he is trained on the job by the nursing staff to take care of promotional, preventive and rehabilitation activities. However, he can benefit from a certificate of recognition [12]. On the other hand, students who have followed a diploma course in community health are therefore fully-fledged health professionals like doctors, nurses, midwives and also participate in improving the health of populations. It is a new body recognized by the country's civil service ministry. Their function is to support the communities by transferring their knowledge and skills to them so that they can fully engage in the resolution of their health problems. [13-15].

Before the advent of initial training in community health, community health activities were carried out by healthcare professionals (doctors, nurses, etc.). The latter benefited from capacity building in the field of community health during training. This is essentially continuous training (specialization in public health, etc.). [16]. However, these healthcare professionals were more concerned with healing and management activities, which is why they relied on CHWs for community health activities. Thus, it is recognized that these CHWs have played an important role in the health system by contributing, for example, to the reduction of maternal and infant morbidity and mortality. In fact, in Africa, community health worker programs have experienced a renaissance over the past two decades, to such an extent that many countries are proud to have such a program nationally. [17].

Thus, this initial training in community health fills a deficit in the health system, that is to say the failure to efficiently take into account health promotion and prevention activities, but also the involvement of communities. It is important that the community (healthcare staff and population and NGOs) and decision-makers can differentiate between "community health workers" and "community health professionals" for a better integration of all actors in the system. We may wonder whether we should not change the term «community health workers' as "community health actors" as in Senegal to avoid this confusion. In Senegal, the number of Community Health Actors was 26,847 in 2017 with two categories of actors who are community care actors (ACs) and community promotion 
and prevention actors (ACPP) [18]. In addition, the outbreak of Ebola virus disease (EVD) [19] in 2014-2015, and Corona virus diseases in 2020 demonstrated the importance of the role of communities in the resilience of health systems and in preparing for health crises. Only a community response and beyond community health could contribute to lasting solutions.

\subsection{Community Health Approach or Public Health}

Community health according to the WHO definition [20] requires the participation and commitment of community members in the management of their individual and collective health. Moreover decision-making emanates from the community and not from health professionals who, moreover, must support this community in choosing the strategies to be adopted. Health professionals become resource people for the community. Thus, they must have for finality the emancipation of the populations with which they intervene, the gradual formation of their capacity to take charge of their own health individually and collectively. They can achieve this through structured activities, integrated into a social development project, and based on their full participation $[13,20]$. Therefore, community health presupposes the involvement, participation, commitment and ownership by this same community of the strategies implemented with a view to their sustainability. However in our context, it has been noted that decision making until now, it is generally up to the healthcare professionals, that the community is only involved when the activities are implemented, that is to say that it is only informed, that the funding is not endogenous but depends on the partners and that the CHWs are trained to carry out the activities and that there is no ownership of the project or activities by the community. It is therefore an approach that relates more to public health defining plans and programs at the central level, rather than community health involving populations in the management of their health. The mobilization of community actors (community intermediaries, community health workers, etc.) in a program does not make it a community health program since they are only trained to carry out activities. $[21,22]$. It should be noted that the community is at the center of these different concepts of public health, primary health care, community health, health promotion, hence the importance of clarifying them and showing the boundaries between them benefit health professionals and decisionmakers so that all speak the same language.

\subsection{The Emergence of Jurisdictional Conflicts}

In the private sector, in particular at the NGO level, community health graduates are recruited on the basis of a job description. The purpose of a job description is to describe in detail the roles, missions and real responsibilities assigned to each job. So there is almost no conflict of jurisdiction.

On the other hand, in the public sector, community health graduates most often work at the level of medical regions or health districts or they often occupy the position of "supervisor or focal point for community health". The person in charge of this position is a member of the regional "ECR" team or the "ECD" district management team. [10] Including among others the regional or district chief doctor, the president of the health committee, other supervisors or focal points (mother and child health, primary health care, health education...)..... It was found that the missions of supervisors or focal points in "community health, primary health care and health education" are almost similar or even identical. In the context of a restructuring or reorganization of the medical region or health district, it would be advisable to merge these three positions and make one position that could be assigned to a community health professional with a diploma in Master.

\subsection{The Status of the Implementation of the Community Health Policy}

Our health authorities are aware that the resolution of health problems can only be done with community involvement; hence the establishment of a community health unit [10]. A better knowledge of the determinants of health by the community is a major asset. However community health should not be a strategy intended primarily for the base of the health pyramid (health huts and posts) and for the rural world. It must concern all the structures of the health pyramid from the health hut (basic level) to the national hospital (highest level of care). Indeed, the hospital concentrates most of the resources (human, financial, logistical) and skills, hence the importance that it is in the community health system.

Health centers and hospitals also have the role of individual and collective protection of populations, which is why they must integrate, in addition to their traditional activities (support), other promotional, preventive, rehabilitation and health activities. support at the end of life (chronic diseases). Community health should also focus on urban populations since, according tothe World Health Organization, nearly two-thirds of the world's population will live in cities by 2030; Africa and Asia will be home to $70 \%$ [23].

However, today, urban health is also characterized by the coexistence communicable diseases (overcrowding, poor hygiene, pollution, etc.) and non-communicable diseases (change in lifestyle, smoking, traffic accident, etc.). The majority of the population will be poor and will live in slums, which pose a huge challenge to equitable and sustainable development, hence the interest of community health. This community health will be based on two essential elements which are the basis of city policy: partnership and participation of residents [24].

It is important to promote preventive and rehabilitation promotional activities by integrating them into the information system like curative activities such as morbidity and mortality. 


\section{Conclusion}

The goal of community health training is to train professionals with knowledge and skills enabling them to contribute to improving the health of populations. It is an innovative but also professional training alternating theory and field practice. Indeed community health is a local approach to solving a community's health problems involving their active participation at all stages. It is theoretically implemented by a group bringing together professionals and the population. However, this concept of community health does not refer to a univocal definition but to a wide variety of realities. Community health professionals are most closely assimilated to community health workers. In addition, they will have to integrate a health system focused more on curative care and leaving little room for prevention and health promotion. Regarding the community health approach, it is more oriented towards rural populations and at the base of the health pyramid (health posts and huts) whereas it must be global (urban environment, hospitals, etc.) and be based on a community engagement with community development as its goal. The establishment of a policy and a strategic plan for community health reflects the option of our decisionmakers to involve the populations in all development programs. But all actors (professionals, decision-makers, populations, and partners) must have the same understanding of the concept of community health, its approach and its strategies.

\section{Recommendations}

1. Clarify the concept of community health to health professionals, decision-makers and partners,

2. Recruit community health professionals at the level of the Ministry of Health and Social Action

3. Propose a restructuring of the composition of district management teams by integrating community health professionals

4. Integrate promotional and prevention activities into the information and data collection system

5. Organize "open house" days for greater visibility of community health training.

\section{Conflicts of Interest}

All the authors do not have any possible conflicts of interest.

\section{Source of Funding}

Supporting research work. The sources of funding are those coming from Unicef Senegal and the UFR SDD of the $\mathrm{UADB}$.

\section{Acknowledgements}

Thanks to the Unicef Senegal team, to the Health and
Nutrition Research Team of UFR SDD of UADB and to the Health and Transitions in Africa Research Team of the International Joint Unit 3189 "Environment, Health and Societies".

\section{References}

[1] Decree 2004-916 on "creation and organization of a CUR in Bambey, www.uadb.edu.sn/index.php/presentation/historique.

[2] WHO: The community health worker, Practical guide: Guidelines for training Guidelines for adaptation, Geneva 1987.

[3] A Crismer, JL Belche, J LV Vennet. Primary health care, more than primary care. Public health 2016; 3 (28): 375-379.

[4] Sandrine Mohamed. What is community health? An example of a participatory and multisectoral approach in a municipality in the Canton of Geneva, Switzerland. Psychiatric information 2015; 7 (91): 563 to 567. https://www.cairn.info/revue-1information-psychiatrique-2015-7-page-563.htm.

[5] Renaudot Institute. Community health practice: from intention to action. Lyon: Éditions Chroniques Sociales 2001.

[6] Carole Dane. Empowerment, a concept for France? Social life 2007; 2 (2): 59-72 www.cairn.info >social-life-review-2007-2page-59

[7] Marie-Hélène Bacqué, Carole Biewener. Empowerment, a new vocabulary for talking about participation?. Economic and social ideas 2013; 3 (173): 25-32. https://www.cairn.info/revue-idees-economique-et-sociales2013-3-page-25.html.

[8] Vanderveken Juliette. The community approach: a methodology that promotes health from social to town planning, including justice... all concerned!. Health Education, Number 343 April 2018.

[9] Ministry of Health and Social Action, General Directorate of Health, Community Health Unit, December, National Strategic Plan for community health 2014. www.sante.gouv.sn >sites> default > files> planssantcomun.

[10] Health pyramid of the Ministry of Health and Social Action http://www.sante.gouv.sn/politique-de-sante/pyramide-desant $\% \mathrm{C} 3 \% \mathrm{~A} 9$.

[11] Lawrence A. Palinkas, P, Sarah M. et al. Purposeful sampling for qualitative data collection and analysis in mixed method implementation research Adm Policy Ment Health. 2015; 42 (5): $533-544$. https://www.ncbi.nlm.nih.gov/pmc/articles/PMC4012002/.

[12] Marie Jaisson.The study of medical practices: the screen of the sociology of professions. File Saude soc 2018; 27 (3). https://www.scielosp.org/article/sausoc/2018.v27n3/704$714 / \mathrm{en} /$.

[13] Kachi Odile Angbo-Effi, Damus Paquin Kouassi, Gnissan Henri Auguste Yao et al. Factors determining the consumption of street drugs in urban areas. Public health 2011; 6 (23): 455464. https://www.cairn.info/revue-sante-publique-2012-2page-165.htm.

[14] Karl Blanchet. Community health, an essential issue. Diplomatic world April 2016. 
[15] Mukalenge Chenge, Jean Van der Vennet, Denis Porignon: The health map of the city of Lubumbashi, Democratic Republic of Congo Part I: problematic of health coverage in Congolese urban areas Global Health Promotion 2010; 17 (3). https://doi.org/10.1177/1757975910375173.

[16] https://www.ised.sn/index.php/les-formations/le-master-desante-publique.

[17] Jourdan D., O'Neill M., Dupéré S. et al. Forty years later, where is community health?. Public Health 2012; 24 (2): 165178 .

[18] Directorate General of Health Community Health Unit, national strategic plan for community health 2014-2018. www.sante.gouv.sn/sites/default/files/BAT F Elected guide.

[19] Mbaye EM, Kone S, Ka O et al. Evolution of community involvement in the response to Ebola. Public Health 2017; 29 (4): 487-496.

[20] WHO, Community health worker programs in the African region: evidence and options, guidance note, WHO Regional Office for Africa 2017. www.afro.who.int/sites/default/files/ 2017- 07.

[21] Erkki Vartiainen. North Karelia Project. Prevention of cardiovascular disease in Finland. Glob Cardiol Sci Pract 2018 (2): 13 .

[22] Sandrine M. What is community health? An example of a participatory and multisectoral approach in a municipality in the Canton of Geneva, Switzerland. Psychiatric information 2015; 7 (91): 563-567.

[23] United Nations Development Program. Strategy in support of sustainable urbanization UNDP support for sustainable, inclusive and resilient cities in a developing world 2016. www.undp.org/content/dam/undp/library/Sustainable.

[24] Pagani V, Kivits J, Minary L, et al. Complexity. concept and challenges for public health interventions. Public Health 2017; 29 (1): 31-39. 